In Crescendo, 2020; 11(1): 95-103

Fecha de recepción: 26/02/2020

Fecha de aceptación: 28/03/2020

\title{
FORMALIZACIÓN DEL EMPLEO Y SU IMPACTO EN EL DESEMPEÑO LABORAL DE LOS TRABAJADORES EN EL DISTRITO DE CHIMBOTE, 2018-2019
}

\author{
FORMALIZATION OF EMPLOYMENT AND ITS IMPACT ON \\ THE LABOR PERFORMANCE OF WORKERS IN \\ THE CHIMBOTE DISTRICT, 2018-2019
}

\author{
Francisco Carruitero ${ }^{1}$, Héctor Martínez 2 , Tula Benites ${ }^{3}$, \\ Víctor Rojas Lujan ${ }^{4}$, Guillermo Medina ${ }^{5}$
}

\section{RESUMEN}

El propósito de la investigación fue estudiar la formalización del empleo y su impacto desempeño laboral de los trabajadores en el Distrito de Chimbote, 2018-2019. El tipo de estudio es no experimental, correlacional causal transversal y los métodos de investigación aplicados fueron el inductivo, deductivo, el descriptivo y estadístico. La muestra fue determinada por 50 trabajadores informales y 50 trabajadores del sector formal, mediante el muestreo estadístico no probabilístico. Se utilizó la técnica de la encuesta con un cuestionario tipo escala de Likert. El análisis de la información se realizó empleando la estadística descriptiva e inferencial, como herramientas para el procesamiento de los datos se utilizó el programa estadístico SPSS, versión 23.

1 Abogado, Magister en Sociología y Doctor en Derecho (Revalida) por la Pontificia Universidad Católica del Perú. Profesor ordinario en la Universidad Nacional Mayor de San Marcos.

2 Abogado, Magister en Derecho Civil y Comercial por la Universidad Nacional Mayor de San Marcos. Profesor Principal en dicha casa de estudio..

3 Abogada por la Universidad Nacional de Trujillo, Doctora en Derecho Constitucional por la Universidad Privada Antenor Orrego de Trujillo, profesora ordinaria en dicha casa de estudio.

4 Abogado, Magister y Doctor en Derecho por la Universidad Nacional de Trujillo, Profesor Principal en la Universidad Nacional de Tumbes.

5 Abogado por la Universidad Privada San Pedro, Magister en Derecho Penal y estudios de doctorado en Derecho por la Universidad Nacional de Trujillo. 
Se concluye que existe un impacto significativo, de la formalización del empleo en el desempeño laboral de los trabajadores en el Distrito de Chimbote, 2018-2019; según el coeficiente de contingencia del estadístico de prueba Tau-b de Kendall el valor es $\tau=0,845$, con nivel de significancia menor al 5\% de significancia estándar $(\mathrm{P}<0,005)$. Así mismo se obtuvo como resultado que el $40 \%$, de los trabajadores formales tienen un muy alto desempeño laboral, alto $20 \%$, medio $20 \%$, bajo $12 \%$ y muy bajo $8 \%$. Y los trabajadores informales en su desempeño laboral tienen un nivel muy alto el $10 \%$, alto $10 \%$, medio $20 \%$, bajo $40 \%$ y muy bajo $20 \%$.

PALABRAS CLAVE: Formalización laboral, informalidad, empresas metalmecánicas.

\section{ABSTRACT}

TThe purpose of the research was to study the formalization of employment and its impact on the labor performance of workers in the Chimbote District, 2018-2019. The type of study is non-experimental, cross-causal correlational and the applied research methods were inductive, deductive, descriptive and statistical. The sample was determined by 50 informal workers and 50 formal sector workers, through non-probability statistical sampling. The survey technique was used with a Likert scale questionnaire. The analysis of the information was carried out using descriptive and inferential statistics. The statistical program SPSS, version 23 was used as tools for data processing. It is concluded that there is a significant impact of job formalization on the job performance of workers in the Chimbote District, 20182019; according to the contingency coefficient of the Kendall Tau-b test statistic, the value is $\tau=0.845$, with a significance level of less than $5 \%$ of standard significance $(\mathrm{P}<0.005)$. Likewise, it was obtained as a result that $40 \%$ of formal workers have a very high job performance, high 20\%, medium 20\%, low 12\% and very low $8 \%$. And informal workers in their job performance have a very high level of 10\%, high 10\%, medium $20 \%$, low $40 \%$ and very low $20 \%$.

KEY WORDS: Labor formalization, informality, metalworking companies.

\section{INTRODUCCIÓN}

Según el Instituto Nacional de Estadística e Informática (INEI) en el Perú unas 239,000 personas obtuvieron un empleo informal entre abril del 2018 y marzo del 2019. Esto contrasta con las 98 mil personas que sí obtuvieron un empleo formal durante el mismo periodo de tiempo en el país. En los nuevos empleos formales: en el área urbana: 35 mil son para personas entre 14 y 24 años de edad, 28,000 para personas entre 25 a 44 años de edad y 34,000 corresponden a personas mayores a 45 años. Los nuevos empleos informales. En los nuevos empleos informales: 150 mil personas mayores a 45 años, 118,000 entre 25 y 44 
años, 30,000 entre las personas de 14 y 24 años. Así, el empleo formal en el país se mantiene en $27,4 \%$ a nivel nacional, mientras que el empleo informal se ubica en $72,6 \%$. En el 2018, la población ocupada se incrementó en 324,000 personas. Sin embargo, 5 mil personas entre 14 y 24 años dejaron de estar ocupadas. Otras 130 mil personas de 25 a 44 años se incorporaron a la población ocupada, mientras que otras 200 mil mayores de 45 años también lo hicieron. La tasa de desempleo se redujo en 0,2\% a nivel nacional durante el trimestre móvil enero-febreromarzo del 2019. (Diario Gestión, 2019)

El concepto de informalidad fue acuñado por (Hart, 1970), economista de la Organización Internacional del Trabajo, sustentándose en Ghana y un informe sobre Kenya. "En dicho informe se encontró que las personas pobres lograban sobrevivir mediante oficios, actividades y tareas que se realizaban a pequeña escala y -sin ser actividades delictivas- a menudo se encontraban al margen de las normativas vigentes. Observaron que el sector informal incluye un amplio rango de actividades: desde aquellas vinculadas a la sobrevivencia hasta otras de negocios rentables, pero en muchos casos eran actividades no reconocidas, no registradas, no protegidas y no reguladas"

El término empleo informal ha sido definido por muchos especialistas, (Tokman, 1978), (De Soto, 1986) ; (Carbonetto, et al, 1984), entre otros, pero los autores de este trabajo resaltan el estudio de (Loayza, 2013), quien desde una perspectiva legalista de la informalidad, estudia sus causas y consecuencias y luego aplica el análisis al caso del Perú en particular. Esta investigación se inicia con una discusión de la definición y las medidas de la informalidad. Afirma que las razones por las que una informalidad generalizada representa una gran preocupación. Se analiza los principales determinantes de la informalidad, se sostiene que ella aparece cuando se combinan una excesiva carga de regulaciones e imposiciones, unos servicios públicos deficientes y una débil capacidad de monitoreo y sanción por parte del Estado. Esta combinación resulta especialmente explosiva para un país que sufre de bajos niveles de educación, presiones demográficas y estructuras de producción primarias. (Loayza, 2007)

Así mismo diversos autores asumen una gran variedad de definiciones de formalización del empleo, para (Jaramillo, 2013); (Díaz, 2014); (Haltiwanger, Jarmin y Miranda (2013); (Tello, 2015), para los autores de este trabajo resalta la investigación de (Chacaltana y Salazar 2018) y sus planteamientos se pueden resumir en lo siguiente: en las últimas décadas es el proceso de formalización del empleo se debe a las transformaciones en los mercados de trabajo de América 
Latina, se estima que de los 51 millones de empleos que se crean en la región en el decenio 2005-2015, 39 millones son empleos formales, lo cual permite reducir la tasa de empleo informal en este periodo. Diversos factores contribuyen en este proceso: un periodo de crecimiento económico excepcional, transformaciones económicas y sectoriales importantes y políticas deliberadas y explícitas hacia la formalización. A pesar de estos avances, el nivel de informalidad es aún elevado en una región en la que muchos países son de renta media. Y a esto hay que sumarle nuevos desafíos asociados a las nuevas tendencias del futuro del trabajo, que traen al mismo tiempo nuevas formas de informalidad y nuevas formas de formalidad".

En el Perú, el Ministerio de Trabajo, a través de la Resolución Ministerial 071-2018-TR se aprobó: "La Estrategia Sectorial para la Formalización Laboral 2018-2021", el cual tiene por finalidad enfrentar la informalidad laboral como problema estructural el cual afecta negativamente el crecimiento económico, la productividad laboral y el bienestar social y dificulta el acceso al crédito al reducir la recaudación tributaria. La informalidad permite la exclusión del acceso a la protección de la legislación laboral y de la seguridad social por parte de los trabajadores que se encuentran en dicha situación y no permite un empleo digno.

Para (Belapatiño, et al, 2017), en el Perú, la informalidad laboral se ha reducido en los últimos años, sin embargo, aún se mantiene en niveles elevados, lo que hace necesario tomar acciones para reducirla y disminuir sus efectos perniciosos. Se requiere mejorar la normatividad del mercado de trabajo en Perú para subsanar los elementos que inhiben la contratación formal. Es importante la fiscalización laboral y elevar la productividad de los trabajadores.

Entre los factores que usualmente se consideran causas de la informalidad laboral son; una legislación que, directa o indirectamente, desincentiva la contratación formal, es rígida, salarios mínimos; La débil fiscalización laboral que disminuye la probabilidad de que los infractores sean penalizados por no cumplir con la legislación; La baja productividad de los trabajadores que no hace rentable su contratación bajo las condiciones que la ley exige; la percepción de que los beneficios de ser un trabajador formal no son lo suficientemente atractivos y la Insuficiente demanda laboral en el sector formal de la economía. (Belapatiño, et al, 2017)

La fuerza laboral formal es el factor clave dentro de una empresa, para el logro de los objetivos estratégicos de esta. A medida que su personal sea formal permitirá que las empresas tengan acceso al crédito, obtener mayor rentabilidad 
de tal manera que la formalización del empleo permitirá mayores beneficios a la empresa y los trabajadores.

El problema de los trabajadores del distrito de Chimbote se encuentra que son informales, carentes de una legislación que los proteja, salarios por debajo del mínimo, débil fiscalización laboral, baja productividad y una baja percepción del sector formal.

En esta investigación la interrogante a resolver fue la siguiente: ¿Cuál es el impacto de la formalización del empleo para mejorar el desempeño laboral de los trabajadores en el Distrito de Chimbote, 2018-2019? Se planteó como objetivo general determinar el impacto de la formalización del empleo para mejorar el desempeño laboral de los trabajadores en el Distrito de Chimbote, 2018-2019.

\section{MÉTODOS}

La investigación corresponde a un estudio descriptivo correlacional. La unidad de análisis estuvo conformada por 48 trabajadores informales y 48 trabajadores formales metal mecánicas del distrito de Chimbote. Estos datos se obtuvieron de la aplicación de nuestra encuesta.

La muestra estuvo conformada por 100 trabajadores, 50 trabajadores informales y 50 trabajadores formales, los cuales fueron seleccionados aplicando el muestreo no probabilístico. La técnica de recolección de información fue la encuesta. El instrumento fue el cuestionario. La validez se efectuó mediante juicio de expertos. La confiabilidad se realizó a través del Coeficiente de Tau-b de Kendall y se procesó mediante el software estadístico SPSS V.23.

\section{RESULTADOS Y DISCUSIÓN}

Los resultados fueron analizados en función a los objetivos propuestos de la investigación. Para recolectar la información, se aplicó un cuestionario, del cual se obtuvieron los datos relacionados con las variables y sus dimensiones. Asimismo, el análisis de los resultados se puede evidenciar en las tablas estadísticas.

En la tabla 1, se observa que los trabajadores formales a los cuales se les realizó el estudio, desarrollan su desempeño laboral tienen un nivel muy alto el 40\%, alto $20 \%$, medio $20 \%$, bajo $12 \%$ y muy bajo $8 \%$. Y los trabajadores informales en su desempeño laboral tienen un nivel muy alto el 10\%, alto 10\%, medio $20 \%$, bajo $40 \%$ y muy bajo $20 \%$. 
Tabla 1

COMPARACIÓN DE LOS PUNTAJES OBTENIDOS ENTRE TRABAJADORES FORMALES E INFORMALES Y EL DESEMPEÑO LABORAL

\begin{tabular}{lrrrr}
\hline Desempeño laboral & \multicolumn{2}{c}{$\begin{array}{l}\text { Trabajadores } \\
\text { formales }\end{array}$} & \multicolumn{2}{c}{$\begin{array}{c}\text { Trabajadores } \\
\text { informales }\end{array}$} \\
\hline Niveles & $\mathbf{f}$ & $\%$ & $\mathrm{f}$ & $\%$ \\
\hline Muy alto & 20 & $40 \%$ & 5 & $10 \%$ \\
Alto & 10 & $20 \%$ & 5 & $10 \%$ \\
Medio & 10 & $20 \%$ & 10 & $20 \%$ \\
Bajo & 6 & $12 \%$ & 20 & $40 \%$ \\
Muy bajo & 4 & $8 \%$ & 10 & $20 \%$ \\
\hline Total & 50 & $100 \%$ & 50 & $100 \%$ \\
\hline
\end{tabular}

Fuente: Instrumentos aplicados a los trabajadores informales y formales, 2018-2019.

Tabla 2

COMPARACIÓN DE LOS PUNTAJES OBTENIDOS ENTRE TRABAJADORES

FORMALES E INFORMALES Y EL NIVEL DE REMUNERACIONES

\begin{tabular}{lrrrr}
\hline Remuneraciones & \multicolumn{2}{c}{$\begin{array}{c}\text { Trabajadores } \\
\text { formales }\end{array}$} & \multicolumn{2}{c}{$\begin{array}{c}\text { Trabajadores } \\
\text { informales }\end{array}$} \\
\hline Niveles & $\mathbf{f}$ & $\%$ & $\mathrm{f}$ & $\%$ \\
\hline Muy alto & 25 & $50 \%$ & 5 & $10 \%$ \\
Alto & 10 & $20 \%$ & 5 & $10 \%$ \\
Medio & 5 & $10 \%$ & 10 & $20 \%$ \\
Bajo & 5 & $10 \%$ & 10 & $20 \%$ \\
Muy bajo & 5 & $10 \%$ & 20 & $40 \%$ \\
\hline Total & 50 & $100 \%$ & 50 & $100 \%$ \\
\hline
\end{tabular}

Fuente: Instrumentos aplicados a los trabajadores informales y formales, 2018-2019. 
Tabla 3

COMPARACIÓN DE LOS PUNTAJES OBTENIDOS ENTRE TRABAJADORES FORMALES E INFORMALES Y EL PAGO DE TRIBUTOS

\begin{tabular}{lrrrr}
\hline Tributos & \multicolumn{2}{c}{$\begin{array}{c}\text { Trabajadores } \\
\text { formales }\end{array}$} & \multicolumn{2}{c}{$\begin{array}{c}\text { Trabajadores } \\
\text { informales }\end{array}$} \\
\hline Niveles & $\mathbf{f}$ & $\%$ & $\mathbf{f}$ & $\%$ \\
\hline Muy alto & 20 & $40 \%$ & 4 & $8 \%$ \\
Alto & 10 & $20 \%$ & 4 & $8 \%$ \\
Medio & 8 & $16 \%$ & 7 & $14 \%$ \\
Bajo & 6 & $12 \%$ & 20 & $40 \%$ \\
Muy bajo & 6 & $12 \%$ & 15 & $30 \%$ \\
\hline Total & 50 & $100 \%$ & 50 & $100 \%$ \\
\hline
\end{tabular}

Fuente: Instrumentos aplicados a los trabajadores informales y formales, 2018-2019.

Tabla 4

COMPARACIÓN DE LOS PUNTAJES OBTENIDOS ENTRE TRABAJADORES FORMALES E INFORMALES Y SUS NIVELES DE PRODUCTIVIDAD

\begin{tabular}{lrrrr}
\hline Productividad & \multicolumn{2}{c}{$\begin{array}{c}\text { Trabajadores } \\
\text { formales }\end{array}$} & \multicolumn{2}{c}{$\begin{array}{c}\text { Trabajadores } \\
\text { informales }\end{array}$} \\
\hline Niveles & $\mathbf{f}$ & $\%$ & $\mathbf{f}$ & $\%$ \\
\hline Muy alto & 20 & $40 \%$ & 0 & $0 \%$ \\
Alto & 10 & $20 \%$ & 0 & $0 \%$ \\
Medio & 10 & $20 \%$ & 10 & $20 \%$ \\
Bajo & 5 & $10 \%$ & 20 & $40 \%$ \\
Muy bajo & 5 & $10 \%$ & 20 & $40 \%$ \\
\hline Total & 50 & $100 \%$ & 50 & 100 \\
\hline
\end{tabular}

Fuente: Instrumentos aplicados a los trabajadores informales y formales, 2018-2019. 
En la tabla 2, se observa que los trabajadores formales a los cuales se les realizó el estudio, sus remuneraciones tienen un nivel muy alto el 50\%, alto 20\%, medio $10 \%$, bajo $10 \%$ y muy bajo $10 \%$. Y los trabajadores informales en sus remuneraciones tienen un nivel muy alto el $10 \%$, alto $10 \%$, medio $20 \%$, bajo $20 \%$ y muy bajo $40 \%$.

En la tabla 3, se observa que los trabajadores formales a los cuales se les realizó el estudio, sus pagos de tributos tienen un nivel muy alto el 40\%, alto 20\%, medio $16 \%$, bajo $12 \%$ y muy bajo $12 \%$. Y los trabajadores informales en sus pagos de tributos tienen un nivel muy alto el $8 \%$, alto $8 \%$, medio $14 \%$, bajo $40 \%$ y muy bajo $30 \%$.

En la tabla 4, se observa que los trabajadores formales a los cuales se les realizó el estudio, sus niveles de productividad tienen un nivel muy alto el $40 \%$, alto $20 \%$, medio $30 \%$, bajo $10 \%$ y muy bajo $10 \%$. Y los trabajadores informales tienen su nivel de productividad tienen un nivel muy alto el $0 \%$, alto $0 \%$, medio $20 \%$, bajo $40 \%$ y muy bajo $40 \%$.

\section{CONCLUSIONES}

Se determinó que la formalización del empleo tiene impacto significativo en el desempeño laboral de los trabajadores informales del Distrito de Chimbote, 2018-2019; observando un nivel medio al respecto según el coeficiente de contingencia del estadístico de prueba Tau-b de Kendall el valor es $=0,845$, con nivel de significancia menor al $5 \%$ de significancia estándar $(\mathrm{P}<0,005)$ y un coeficiente de correlación Rho Spearman $=0,905$.

Se observa que el $40 \%$ de los trabajadores formales del distrito de Chimbote desarrollan un nivel muy alto de desempeño laboral; mientras que solamente el $10 \%$ de los trabajadores informales desarrollan un nivel de muy alto nivel. Se aprecia que el 20\% de los trabajadores formales desarrollan un alto nivel de desempeño laboral, mientras que el 10\% de los trabajadores informales desarrollan un nivel alto. Se conoce que el 20\% de los trabajadores formales tiene un nivel medio, mientras que el 20\% de trabajadores informales tienen también un nivel medio. El 12\% de los trabajadores formales tienen nivel bajo de desempeño laboral y el $40 \%$ de trabajadores informales tienen un nivel bajo de desempeño laboral. El 8\% de trabajadores formales tienen un desempeño laboral muy bajo, mientras que el $20 \%$ de los trabajadores tienen un nivel muy bajo de desempeño laboral. 
AGRADECIMIENTO a la Universidad Nacional Mayor de San Marcos por fomentar este estudio y habernos permitido desarrollar esta investigación.

\section{REFERENCIAS BIBLIOGRÁFICAS}

Belapatiño, V. Grippa, F. y Perea, H., Perú, Informalidad laboral y algunas propuestas para reducirla, Banco Continental, Lima, 2017.

https://www.bbvaresearch.com/wp-content/uploads/2017/01/Observatorio-informalidadlaboral1.pdf

Carbonetto, D, Hoyle, J y Tueros, M., (1984), El Sector Informal, Cedep, Lima.

De Soto, H, Ghersi, E. Ghibellini, M., Instituto Libertad y Democracia (1986), El otro sendero: la revolución informal, El Barranco: Instituto Libertad y Democracia, Lima.

Diario Gestión, (2019), INEI El empleo informal en el país sigue creciendo más que el formal, 2019. https:/gestion.pe/economia/inei-informal-pais-sigue-creciendo-formal-266936-noticia/

Díaz, J. (2014), Formalización empresarial y laboral. En: Infante y Chacaltana. Hacia un desarrollo inclusivo. El caso de Perú, OIT, Lima.

Haltiwanger, J; Jarmin R y Miranda J. (2013), "Who creates jobs? Small versus large versus young. The review of economics and statistics", vol. XCV May.

Hart, K., (1972), Employment, Income and Inequality: A Strategy for Increasing Productive Employment in Kenya, OIT: Ginebra.

Jaramillo, M., (2013), Employment growth and segmentation in Peru, 2001-2011. Country case study on labour market segmentation, OIT: Ginebra.

Loayza, N., (2013), Causas y consecuencias de la informalidad en el Perú, Banco Central de Reserva del Perú, Lima.

Salazar, J. y Chacaltana, J., (2018) "La informalidad en América Latina i el Caribe: iPor qué persiste y cómo superarla?", en Políticas de formalización en América Latina, avances y desafíos, en Salazar y Chacaltana (Coordinadores) OIT, Oficina Regional para América Latina y el Caribe, Lima.

Tello, Mario (2015). ¿Es la informalidad una opción voluntaria o la última alternativa de empleo en el Perú? CIES. Lima.

Tokman, V. E. (1978), "An exploration into the nature of informal-formal sector relationships", World Development, vol. 6, núms.9-10 pp. 1065-1075. 